“ (C) 2018 IEEE. Personal use of this material is permitted. Permission from IEEE must be obtained for all other uses, in any current or future media, including

reprinting/republishing this material for advertising or promotional purposes, creating new collective works, for resale or redistribution to servers or lists, or reuse of any copyrighted component of this work in other works." 


\title{
Spun High Birefringence Bismuth/Erbium Co- Doped Photonic Crystal Fibre with Broadband Polarized Emission
}

\author{
Yanhua Luo ${ }^{1 *}$, Yushi Chu ${ }^{1}$, Kevin Cook$^{2}$, Ghazal Tafti ${ }^{1}$, Shuai Wang ${ }^{1}$, Wenyu Wang ${ }^{1}$, Yuan Tian ${ }^{1}$, John Canning ${ }^{2}$ and Gang-Ding Peng ${ }^{1}$ \\ ${ }^{1}$ Photonics \& Optical Communication, School of Electrical Engineering and Telecommunications, UNSW Sydney, NSW, 2052, Australia \\ ${ }^{2}$ interdisciplinary Photonics Laboratories (iPL), School of Electrical \& Data Engineering, UTS, NSW 2007 Australia \\ email: yanhua.luo1@unsw.edu.au
}

\begin{abstract}
Spun Hi-Bi bismuth/erbium co-doped photonic optical fibre (SHB-BEPCF) has been fabricated using preform stacking and draw tower self-pressurization. Results demonstrate broadband and partially elliptical polarized NIR emission under un-polarized $830 \mathrm{~nm}$ pumping spanning $\Delta \lambda \sim$ $520 \mathrm{~nm}$ centred at $1430 \mathrm{~nm}$.
\end{abstract}

Keywords-Spun PCF, high-birefringence (Hi-Bi), Bi/Er codoped optical fibre, broadband, polarized emission

\section{INTRODUCTION}

Structured optical fibres such as periodic photonic crystal fibres (PCF) and quasi-periodic Fresnel fibres continue attracting interest because of the unique properties possible to be fine-tuned simply by positioning a series of air holes around a core defining an effective index instead of physically altering such index through direct chemical doping. Unique properties include endlessly single mode, improved confinement and enhance linear and non-linear properties and Bessel beam generation to focus light outside of a fibre $[1,2]$ and more. The structure of structured optical fibres are highly flexible in design. By designing proper air hole structures, high birefringence structured and photonic crystal fibres (HiBi PCFs [3]) can easily be achieved. Further, spinning of HiBi PCF preform during drawing leads to spun Hi-Bi fibres that can have elliptical or even circular birefringence [4].

When the core rod is replaced with an active rod doped with dopants such as $\mathrm{Er}^{3+}, \mathrm{Yb}^{3+}, \mathrm{Bi}$ ion and more, spun $\mathrm{Hi}-\mathrm{Bi}$ doped structured fibres can be made. Depending on the polarisation beat length, elliptically or circularly polarized optical light is generated output. For laser beam applications this is particularly important since circularly polarised laser emission will have improved cutting efficiencies over linearly polarized or unpolarised lasers and then reduced the total power required [5]. In addition, $\mathrm{Bi} / \mathrm{Er}$ or $\mathrm{Bi} / \mathrm{Er} / \mathrm{Yb}$ co-doped optical fibres with broadband emission covering O-, E, S-, Cand L-bands are potentially useful for many applications [6, 7]. Here, we report the first fabrication of spun $\mathrm{Hi}-\mathrm{Bi} \mathrm{Bi} / \mathrm{Er}-$ doped photonic crystal fibre (SHB-BEPCF) with broadband and elliptically polarized emission.

\section{EXPERIMENTS}

\section{A. Bi/Er Co-doped Rod}

The Bi/Er co-doped fibre preform is fabricated using insitu doping modified chemical vapour deposition (MCVD) [8]. The preform refractive index profile (Fig. 1) is acquired using a preform analyzer. The preform diameter is $\phi \sim 12.84$ $\mathrm{mm}$, the core diameter is $\phi \sim 1.2 \mathrm{~mm}$ and the numerical aperture of the preform is NA 0.25. Based on doping feeding solution and deposition conditions, the doping concentrations of each gradient are estimated as [Si] 25.95, [P] 0.95, [Ge] $\sim 5.21,[\mathrm{Er}] \sim 0.006,[\mathrm{Al}] \sim 0.094$ and $[\mathrm{Bi}] \sim 0.102$ at\%, respectively, which are in agreement with index profiles of the preform measured.

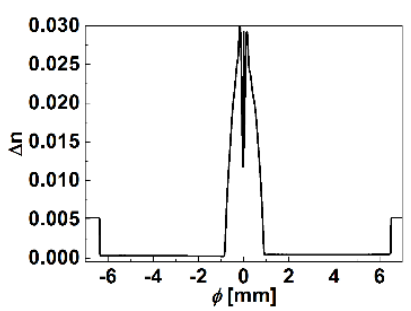

Fig. 1. Refractive index difference, $\Delta$ n, versus radius position, $\phi$, profile of the $\mathrm{Bi} / \mathrm{Er}$ co-doped silica preform.

The doped preform is drawn into the core rod on the fibre drawing apparatus manufactured by Controls Interface. The drawing conditions are: furnace temperature $T_{d}=1920{ }^{\circ} \mathrm{C}$, preform feeding rate $v_{f}=8 \mathrm{~mm}$ and rod drawing rate $v_{d}=0.5$ $\mathrm{m} / \mathrm{min}$. The rod diameter $\phi \sim 2.05 \mathrm{~mm}$ is for the PCF stacked preform shown in Fig. 2a.

\section{B. Hi-Bi Bi/Er Co-doped PCF Preform}

The procedures for fabricating spun Hi-Bi doped PCF can be divided into two stages: preform manufacture and fibre preform drawing. Manufacture includes the structure design, capillary \& rod draw, PCF stacking and preform fusing. The preform is then drawn into fibre on a commercial draw tower.

The PCF design is optimised for given capillary dimensions using Autodesk Inventor and COMSOL Multiphysics software. Ensuring that the entire design is symmetric across the fibre cross-section with no region having any variation in air glass fraction eliminates asymmetric pressure loading during lathe fusing or tower drawing. Deliberate asymmetry in the circular axes is balanced across the core to generate birefringence. The specific design chosen in this work following previous work [4] is a 4-ring Hi-Bi PCF shown in Fig. 2a. It is made up of four rings which numerical simulation suggests has sufficient low loss, $\alpha$, of a few $\mathrm{dB} / \mathrm{km}$ removing the need for additional rings. For the case reported here, slightly thicker capillaries on two sides of the first ring of a regular PCF are used to introduce the form birefringence, effectively altering the propagation constants of the two polarisation eigenstates.

The structured fibre preform is fabricated by stacking capillaries and then inserting into a host tube. Based on the selected design, customised capillaries and rods to be inserted 
into the host tube are drawn with different sizes (Fig. 2b) and placed in a cleanroom for cleaning and removing possible contamination during production.

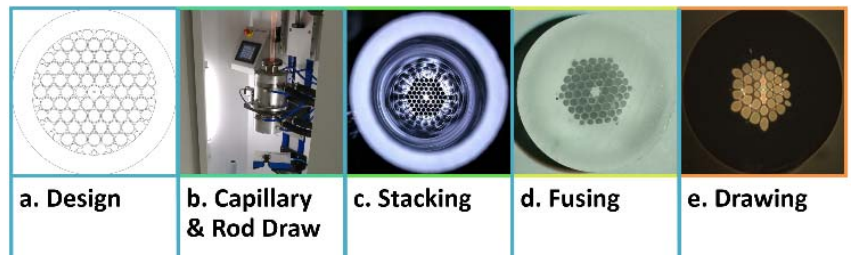

Fig. 2. Course of the fabrication of SHB-BEPCF from the design to final drawing.

Stacking itself is simplified using novel 3D printed holders. These capillaries and rods are then cut into lengths $L$ $\sim 200$ to $300 \mathrm{~mm}$. One end is sealed using high temperature ultraclean ceramic paste. After suitable cleaning of the capillaries, and using the 3D printed hexagonal holder, capillaries are stacked layer by layer into a hexagonal shape. A core rod fabricated by MCVD with $\mathrm{Bi} / \mathrm{Er}$ co-doping is inserted into the centre rather than a normal rod for doped PCF fabrication. Once the hexagonal component is assembled it is held together in place and inserted into the Heraeus host tubing - this approach minimises scratching that would occur if tubes are individually inserted into the host tube and assembled in that fashion. Image of the assembled preform is shown in Fig. 2c.

The stacked preform can be fused on a lathe to remove interstitial holes by placing the interstitial regions under vacuum ( $P \sim$-998 mbar), greatly relaxing the final stage of fibre drawing. This approach is more resilient than direct drawing of the stacked assembly on the tower simply because difficulties managing the differences in hole size between interstitial regions and capillaries is removed altogether. The fusing parameters are: $\mathrm{H}_{2}$ flow rate, scanning rate of the burner and rotating the preform $\left(v_{r} \sim 50 \mathrm{rpm}\right)$ and a fusing temperature (typical $1730{ }^{\circ} \mathrm{C}$ ), all optimised. For further adjustment of the final structure, stretching and sleeving has also been performed. Fig. 2d shows a typical cross section of a fused PCF preform with sufficient fusing for drawing. All the surrounding open regions are fully closed although some interstitial holes between capillaries remain open - these are small enough to not be problematic in drawing. Generally, good symmetric fusing is important to avoid any off-centre spinning during fibre drawing of spun $\mathrm{Hi}-\mathrm{Bi}$ fibres as well as maintaining structure symmetry into fibre form.

\section{Spun Hi-Bi Bi/Er Co-Doped PCF Drawing}

For normal or $\mathrm{Hi}-\mathrm{Bi}$ drawing, the PCF structure is regulated by the furnace temperature, feeding rate, drawing rate as well as the pressure inside the air holes, controlled by purged gas $\left(\mathrm{N}_{2}\right.$ or $\left.\mathrm{Ar}\right)$. When feeding and drawing speeds are fixed, both the structure and dimension of the PCF will be controlled by the drawing temperature and pressure. However, for spun Hi-Bi PCF drawing, it remains a challenge to sustain stable pressure during high speed spinning $\left(v_{s}>50 \mathrm{rpm}\right)$. Therefore, the scheme of the self-pressurization of the air holes is adopted, which means that the structure will be maintained with help from gas pressure sealed inside the preform. The top end of the preform is sealed and the bottom end will be automatically sealed once drawing begins. During drawing, the inner pressure of the preform will slowly achieve a relative balance with the surface tension.
Fig. 3 summarises how the top end of preform can be sealed using three schemes: (1) all the air holes sealed individually at position C; (2) the top of the preform base sealed at position A (large volume existed at the top of the preform) allowing air in different air holes communicating with each other; and (3), the top of the preform sealed at position B with a solid rod (small volume existed at the top of the preform). In scheme 1 , interstitial regions were easily blown up, and the un-balanced pressure between air holes and interstitial region will result in the structure deformation. In scheme 2, the air sealed inside the preform base will generate a high internal pressure with drawing undergoing and destroy the Hi-Bi PCF structure.

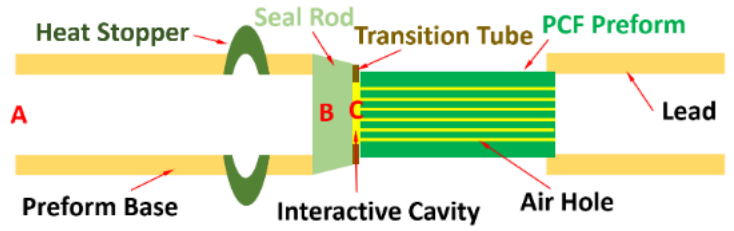

Fig. 3. Schematic diagram of fused PCF preform ready for spun drawing.

Scheme 3 in Fig. 3 is adopted for the fabrication of SHBBEPCF in this work. The preform base, often made of $25 \times 19$ Heraeus quartz tube, allowed the well mounting into the spin feeder of the fibre draw tower. The squeezed bump (heat stopper) stops heat transfer along the preform inside the furnace. A sealing rod at position B was used to seal the top of PCF preform. From the short section of the transition tube ( $2.3 \mathrm{~mm}$, position $\mathrm{C}$ ), the PCF preform is joined to the sealing rod. The interactive cavity will allow the air inside each air holes communicating with each other and achieve a similar internal pressure for different air holes. The lead tube is a short section of the tube, fused with the PCF preform. The lead allows the air inside the air holes ventilating out, and also provides enough weight to start the fibre draw at a lower drawing temperature $\left(T \sim 1855^{\circ} \mathrm{C}\right)$. When the PCF drawing starts, the air holes of the fibre will fully be sealed. Meanwhile, the air sealed inside the air holes will provide enough expansion to balance the collapse due to the drawing and surface tension, maintaining the PCF structure. Once the drawing becomes stable, the preform is spun using the spinner. The spin pitch, $\Lambda$, is determined by the drawing speed and spin velocity.

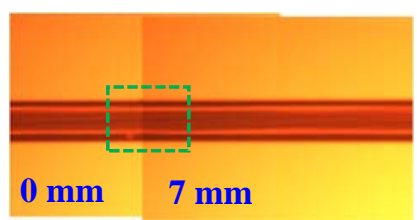

Fig. 4. Side images of SHB-BEPCF taken under the optical microscope, indicating abeat length period of $\sim 7 \mathrm{~mm}$.

The first draw of SHB-BEPCF has been achieved with scheme 3; the cross section is shown in Fig. 2e. The drawing conditions are: furnace temperature $T_{d}=1846{ }^{\circ} \mathrm{C}$, preform feeding rate $v_{f}=0.16 \mathrm{~mm}$, fibre drawing rate $v_{d}=4 \mathrm{~m} / \mathrm{min}$ and spinning rate $v_{s}=600 \mathrm{rpm}$. From these parameters, the physical pitch of the induced helix was $\Lambda \sim 6.7 \mathrm{~mm}$ matched well with that obtained from the images under optical microscope (Fig. 4). This pitch is sufficient to induce elliptical birefringence over long lengths but not sufficient for circular birefringence.

Different from normal Hi-Bi PCF drawing, the drawing time is not long as it is difficult to ensure precise on-axis 
spinning; otherwise, drawing of the fibre is compromised and breaking occurs as the fibre makes contact with dies and coating apparatus further down the draw tower. At higher spinning speeds, axes misalignment leads to more serious problems.

\section{Polarized Emission Measurement}

Using the setup (Fig. 5), the spectral emission of a SHBBEPCF of $70 \mathrm{~cm}$ under $13.1 \mathrm{~mW}$ of $830 \mathrm{~nm}$ un-polarized pumping was measured and shown in Fig. 6. Fig. 6a show the emission spectra when the polarization angle of the polarizer is set at $\theta=240^{\circ}$ (solid line) and $\theta=330^{\circ}$ (dash line), respectively. Fig. $6 \mathrm{~b}$ shows the emission intensity at $1420 \mathrm{~nm}$ vs polarization angle. By comparison of Fig. 6a and 6b, it clearly indicates that the solid line and dash line almost close the maximum and minimum spectra, which is corresponding to the parallel $\left(\theta=240^{\circ}\right)$ and perpendicular $\left(\theta=330^{\circ}\right)$ direction to the optical axis of the output of SHB-BEPCF. The spectrum with the solid line clearly indicates a broad $3 \mathrm{~dB}$ bandwidth over $(1330$ - 1515) nm. The truth bandwidth can be broader if consideration of the spectral compensation to cascaded optical filter, the InGaAs detector and Sigma film polarizer. There is a peak $\lambda=1430 \mathrm{~nm}$ and a shoulder peak at $\lambda \sim 1530 \mathrm{~nm}$ attributed to BAC-Si and $\mathrm{Er}^{3+}$, respectively. The dip at $\lambda \sim 1380 \mathrm{~nm}$ is attributed to $\mathrm{OH}$ absorption.

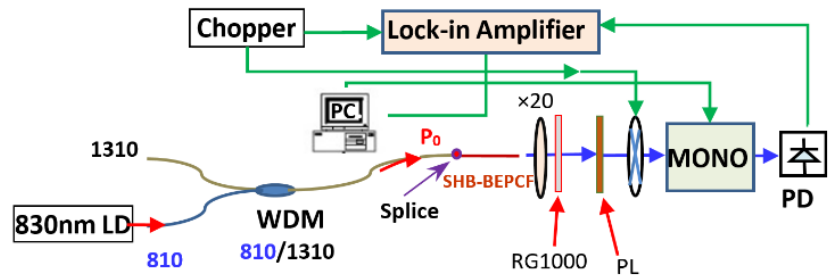

Fig. 5. Experimental setup for the polarized emission measurement. LD laser diode; WDM: wavelength division multiplexing coupler; PC: Computer; MONO: monochromator; PD: photo diode; RG1000: Schott filter RG1000; PL: polarizer (vertical direction is $0^{\circ}$ ).

\section{REUSLT \& DISCUSSION}

The structure of SHB-BEPCF (Fig. 2e) demonstrates that the scheme 3 by self-pressurization maintains the PCF structure well, although the shape has some distortion, such as the fully collapse of $4^{\text {th }}$ ring and Hi-Bi holes. Collapse of the $\mathrm{Hi}-\mathrm{Bi}$ holes demonstrates that the wall thickness of the capillaries used for Hi-Bi holes cannot be too much different from other capillaries for the hexagonal structure stacking. The uneven distribution comes from the asymmetry introduced by the preform processing, especially the sleeving process. Obviously the preform structure design as well as the temperature and length of sealed zone will affect the final fibre structure. Temperature will influence the viscosity and surface tension of silica and also the air pressure in the holes sealed in drawing.

The degree of polarization (DOP) of emission is further studied and evaluated by the following definition [9]:

$$
D O P=\frac{I_{\max }-I_{\min }}{I_{\max }+I_{\min }}
$$

where $I_{\max }$ and $I_{\min }$ are the maximum and minimum emission intensity at certain polarization angle as shown in Fig. 6b. Fig. $6 \mathrm{~b}$ clearly indicates that the emission output from SHBBEPCF is polarized. According to Eq. (1), DOP at $1420 \mathrm{~nm}$ is about 0.33 . Especially, the DOP spectrum can be given by Fig. 7, based on Fig. 6a. Seen from Fig. 7, it shows that the DOP increases with the emission wavelength, which agrees with the trend of the simulation result of the birefringence vs wavelength. Furthermore, the emission output is verified to be partially elliptical polarized light, through the combination measurement with a quarter waveplate and then polarizer.
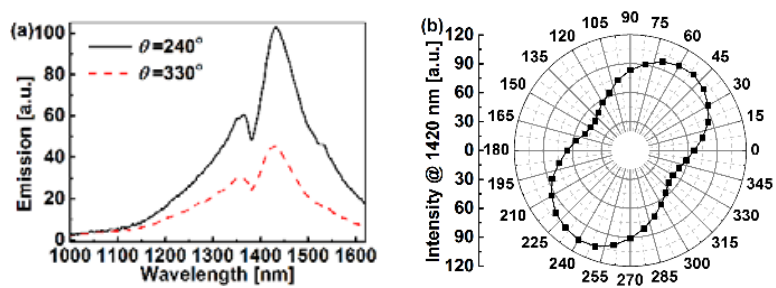

Fig. 6. (a) Maximum $\left(\theta=240^{\circ}\right)$ and minimum $\left(\theta=330^{\circ}\right)$ polarized emission spectra without spectral compensation; (b) Emission intensity at $1420 \mathrm{~nm}$ vs polarization angle.

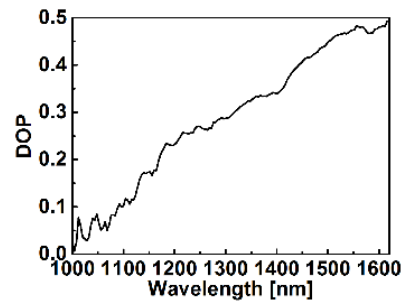

Fig. 7. The degree of polarisation (DOP) spectrum of NIR emission.

\section{CONCLUSION}

In this paper we have successfully demonstrated the first fabrication spun Hi-Bi bismuth/erbium co-doped PCF with spinning pitch length of $\Lambda \sim 6.7 \mathrm{~mm}$. Self-pressurization has been adopted to control the PCF structure. The preliminary result demonstrates that the SHB-BEPCF can produce the broadband and partially elliptical polarized emission under un-polarized $830 \mathrm{~nm}$ pumping. The result also demonstrates that SHB-BEPCF has great potential for elliptically polarized broadband light source.

Acknowledgement: The authors thank the Asian Office of Aerospace R\&D for the grant (FA2386-16-1-4031)

\section{REFERENCES}

[1] J. Canning, E. Buckley, K. Lyytikainen, "Propagation in air by field superposition of scattered light within a Fresnel fibre," Opt. Lett., 28, (4), 230-2332, 2003.

[2] J. Canning, "Fresnel Optics Inside Optical Fibres," in Photonics Research Developments, Chap. 5, Nova Science Publishers, United States, 2008. ISBN 978-1-60456-720-5

[3] H. Kubota, S. Kawanishi, S. Koyanagi, M. Tanaka, and S. Yamaguchi, "Absolutely single polarization photonic crystal fiber," Photon. Technol. Lett., 16, 182-184, 2004.

[4] A. Michie et al., "Spun elliptically birefringent photonic crystal fibre," Opt. Express, 15, 1811-1816, 2007.

[5] J. Canning, "Fibre lasers \& related technologies," Opt. \& Las. In Eng., 44, 647-676, 2006.

[6] Y. Luo, J. Wen, J. Zhang, J. Canning, G.-D. Peng, "Bismuth and erbium co-doped optical fiber with ultra broadband luminescence across O-, E-, S-, C- and L-bands," Opt. Lett., 37, 3447-3449, 2012.

[7] Z. M. Sathi, J. Zhang, Y. Luo, J. Canning, and G. D. Peng, "Improving broadband emission within $\mathrm{Bi} / \mathrm{Er}$ doped silicate fibres with $\mathrm{Yb}$ codoping," Opt. Mater. Express, 5, 2096-2105, 2015.

[8] A. S. Webb et al., "MCVD in-situ solution doping process for the fabrication of complex design large core rare-earth doped fibers," J. Non-Cryst. Solids, 356, 848-851, 2010.

[9] T. Azumi and S. P. McGlynn, "Polarization of the luminescence of phenanthrene," J. Chem. Phys., 37, 2413-2420, 1962. 